\title{
Intensity and physiological responses to the 6-minute walk test in middle-aged and older adults: a comparison with cardiopulmonary exercise testing
}

\author{
E.F. Sperandio ${ }^{1}$, R.L. Arantes ${ }^{2}$, A.C. Matheus ${ }^{1}$, R.P. Silva ${ }^{1}$, V.T. Lauria ${ }^{1}$, M. Romiti ${ }^{2}$, \\ A.R.T. Gagliardi ${ }^{2}$ and V.Z. Dourado ${ }^{1}$ \\ ${ }^{1}$ Departamento de Ciências do Movimento Humano, Laboratório de Epidemiologia e Movimento Humano (EPIMOV), \\ Universidade Federal de São Paulo, Santos, SP, Brasil \\ ${ }^{2}$ Instituto de Medicina Cardiovascular Angiocorpore, Santos, SP, Brasil
}

\begin{abstract}
The 6-minute walk test (6MWT) is a simple field test that is widely used in clinical settings to assess functional exercise capacity. However, studies with healthy subjects are scarce. We hypothesized that the 6MWT might be useful to assess exercise capacity in healthy subjects. The purpose of this study was to evaluate 6MWT intensity in middle-aged and older adults, as well as to develop a simple equation to predict oxygen uptake $\left(\mathrm{V}_{2}\right)$ from the 6-min walk distance (6MWD). Eighty-six participants, 40 men and 46 women, $40-74$ years of age and with a mean body mass index of $28 \pm 6 \mathrm{~kg} / \mathrm{m}^{2}$, performed the $6 \mathrm{MWT}$ according to American Thoracic Society guidelines. Physiological responses were evaluated during the 6MWT using a K4b2 Cosmed telemetry gas analyzer. On a different occasion, the subjects performed ramp protocol cardiopulmonary exercise testing (CPET) on a treadmill. Peak $\dot{\mathrm{V}} \mathrm{O}_{2}$ in the $6 \mathrm{MWT}$ corresponded to $78 \pm 13 \%$ of the peak $\dot{\mathrm{V}} \mathrm{O}_{2}$ during $\mathrm{CPET}_{\text {, }}$ and the maximum heart rate corresponded to $80 \pm 23 \%$ of that obtained in CPET. Peak $\dot{V}_{2}$ in $\mathrm{CPET}_{\text {was adequately }}$ predicted by the 6MWD by a linear regression equation: $\dot{\mathrm{V}} \mathrm{O}_{2} \mathrm{~mL} \cdot \mathrm{min}^{-1} \cdot \mathrm{kg}^{-1}=-2.863+(0.0563 \times 6 \mathrm{MWD})\left(R^{2}=0.76\right)$. The 6MWT represents a moderate-to-high intensity activity in middle-aged and older adults and proved to be useful for predicting cardiorespiratory fitness in the present study. Our results suggest that the 6MWT may also be useful in asymptomatic individuals, and its use in walk-based conditioning programs should be encouraged.
\end{abstract}

Key words: Exercise; Walking; Gas exchange

\section{Introduction}

Cardiopulmonary exercise testing (CPET) provides an accurate assessment of physiological responses induced by exercise and is extremely important in the clinical environment, especially for evaluating peak oxygen uptake $\left(\dot{\mathrm{V}} \mathrm{O}_{2}\right)$. Despite the confirmed validity of CPET, its application remains complex, expensive, and dependent on highly trained professionals. Furthermore, CPET may not be well tolerated by very elderly patients or those with comorbidities. Moreover, CPET is not representative of functional capacity in real life (1). Accordingly, simple and inexpensive ways for assessing aerobic exercise capacity should be investigated that are complementary to CPET.

The 6-min walk test (6MWT) is a simple field walking test that is widely used in clinical settings to assess functional exercise capacity. A great advantage of the 6MWT is its operational simplicity. The 6-min walk distance (6MWD) is often used as an index of cardiorespiratory fitness and has been validated in several populations of patients with chronic diseases (2-4). However, there is little information regarding intensity and physiological responses to field walking tests in healthy individuals, especially in middle-aged adults. Accordingly, we hypothesized that the 6MWT might also be used as a test of sufficient intensity to assess aerobic capacity and prescribe walking programs for health promotion in middle-aged adults and seniors without chronic disease. Therefore, we aimed to evaluate the intensity of 6MWT in middle-aged and older adults and to develop a simple equation to predict their oxygen uptake $\left(\dot{\mathrm{V}} \mathrm{O}_{2}\right)$ from the 6MWD.

Correspondence: E.F. Sperandio: <evandrosperandio@yahoo.com>.

Received July 12, 2014. Accepted November 11, 2014. First published online February 24, 2015. 


\section{Material and Methods}

\section{Participants}

We conducted a cross-sectional study in 86 participants selected from the EPIMOV Study (Epidemiological Study of Human Movement and Hypokinetic Diseases) and enrolled 40 men and 46 women from 40 to 74 years of age. The Universidade Federal de São Paulo Ethics Committee approved the study, and all participants provided written informed consent.

We recruited a convenience sample. Participants who required a walking aid, had abnormal post-bronchodilator lung function (5), or reported having cardiorespiratory, metabolic, neuromuscular, or musculoskeletal disease were excluded from the study.

The participants completed a physical activity readiness questionnaire (PAR-Q) (6) consisting of seven (yes/no) questions about key risk factors for events during exercise. If the participant answered "yes" to one or more questions, then he/she was carefully evaluated by a physician before starting the exercise protocol. A face-to-face interview based on the main cardiovascular risk factors and the physical activity level was also performed. Patients underwent spirometric testing and anthropomorphic measurements were recorded.

\section{Anthropometrics}

Body mass $(\mathrm{kg})$ and stature $(\mathrm{m})$ were measured with participants wearing light clothing and no shoes. The body mass index $\left(B M I, \mathrm{~kg} / \mathrm{m}^{2}\right)$ was then calculated.

\section{Spirometry}

Spirometry was performed using a hand-held spirometer (Quark PFT, Cosmed, Italy) according to the criteria established by the American Thoracic Society (ATS) (5). Forced expiratory volume in $1 \mathrm{sec}\left(\mathrm{FEV}_{1}\right)$, forced vital capacity (FVC), and the $\mathrm{FEV}_{1} / \mathrm{FVC}$ ratio were measured.

\section{MWT}

The 6MWT was performed according to ATS standards (7) at 30-min intervals. The participants were instructed to walk as far as possible for 6 min in a $30-\mathrm{m}$ hallway and were given standardized verbal encouragement every minute. 6MWDs were recorded in meters and as a percentage of reference values for further analysis (8). Before and after the test, the rate of perceived exertion (dyspnea and leg fatigue) was measured using the Borg CR10 scale (9).

Throughout the 6MWT, expired gases were collected and analyzed with a commercially available portable telemetric gas analyzer (K4b2, Cosmed). The gas analyzer, which weighs a total of less than $1 \mathrm{~kg}$, was attached to the participants by shoulder straps, allowing free movement with no discomfort. The manufacturer's recommendations were followed with regard to room air, reference gas, 3-L syringe, and delay calibrations.

The following variables were measured at the peak of the 6MWT: pulmonary oxygen uptake $\left(\dot{\mathrm{V}} \mathrm{O}_{2}\right)$, carbon dioxide production $\left(\dot{\mathrm{V}} \mathrm{CO}_{2}\right)$, heart rate $(\mathrm{HR})$, and minute ventilation ( $\dot{V} E)$. Data were averaged every $15 \mathrm{~s}$.

\section{Cardiopulmonary exercise testing}

Cardiopulmonary exercise testing (CEPT) was performed on a treadmill (ATL, Imbramed, Brazil) on a different day. The laboratory temperature was standardized at $22^{\circ} \mathrm{C}$. Participants were instructed to avoid vigorous physical activity on the day prior to the test, to avoid caffeine and other stimulants, and heavy foods, only on the day of the test, and to wear suitable clothing. After $3 \mathrm{~min}$ of rest, the subjects were tested using a ramp protocol with individualized increases in velocity and inclination until exhaustion according to predicted peak $\dot{\mathrm{V}} \mathrm{O}_{2}$.

Ventilatory, cardiovascular and metabolic responses were continuously monitored during the CPET using a gas analyzer (Quark PFT, Cosmed). The following variables were collected and analyzed: heart rate (HR), oxygen uptake $\left(\dot{\mathrm{V}} \mathrm{O}_{2}\right)$, carbon dioxide production $\left(\dot{\mathrm{V}} \mathrm{CO}_{2}\right)$, and expired minute volume ( $\dot{\mathrm{V}} \mathrm{E})$. The average $\dot{\mathrm{V}}_{2}$ obtained in the last $15 \mathrm{~s}$ of the test was considered peak $\mathrm{VO}_{2}$. Oxygen uptake at lactate threshold $\left(\dot{\mathrm{V}}_{2} \mathrm{LT}\right)$ was estimated using both the gas exchange $\mathrm{V}$-slope and ventilatory techniques, as previously described (10).

\section{Statistical analysis}

Multiple linear regression analysis was used to evaluate the association between 6MWD and CPET at peak $\dot{\mathrm{VO}}_{2}$ adjusted for the influence of age, gender and BMI. Following the multiple regression analysis, we determined that the above covariates did not significantly modify the coefficient of determination $\left(R^{2}\right)$. In this context, the results are presented as a simple linear regression using the peak $\dot{\mathrm{VO}}_{2}$ from CPET and the 6MWD as an outcome predictor. The intensity of the 6MWT was assessed using the $t$ test to compare peak $\mathrm{HR}$ and peak $\mathrm{VO}_{2}$ in the $6 \mathrm{MWT}$ with peak HR and peak $\dot{V}_{2}$ in CPET. Unless otherwise specified, 2-tailed $\mathrm{P}$-values $<0.05$ were considered statistically significant.

Table 1. General characteristics of the study sample $(n=86)$.

\begin{tabular}{lc}
\hline Age (years) & $54 \pm 10$ \\
Gender (\%; M/F) & $46.6 / 53.4$ \\
Weight (kg) & $76 \pm 19$ \\
Height $(\mathrm{cm})$ & $1.67 \pm 0.10$ \\
BMI $\left(\mathrm{kg} / \mathrm{m}^{2}\right)$ & $27 \pm 5$ \\
FVC (\% pred.) & $96 \pm 12$ \\
FEV $(\%$ pred.) & $94 \pm 13$ \\
FEV $1 /$ FVC (\%) & $81 \pm 6$ \\
6 MWD (m) & $608 \pm 97$ \\
6MWD (\% pred.) & $106 \pm 13$ \\
\hline
\end{tabular}

Data are reported as means $\pm \mathrm{SD}$. BMI: body mass index; FVC: forced vital capacity; FEV1: forced expiratory volume in $1 \mathrm{~s}$; 6MWD: distance walked in 6-min walk test. 
Table 2. Intensity of the 6-min walk test (6MWT) considering pulmonary oxygen uptake and heart rate obtained from cardiopulmonary exercise testing (CPET).

\begin{tabular}{lcc}
\hline & CPET & 6MWT \\
\hline Peak $\dot{\mathrm{V}} \mathrm{O}_{2}(\mathrm{~mL} / \mathrm{min})$ & $2662 \pm 885$ & $1618 \pm 543$ \\
Peak $\dot{\mathrm{V}} \mathrm{O}_{2}\left(\mathrm{~mL} \cdot \mathrm{min}^{-1} \cdot \mathrm{kg}^{-1}\right)^{*}$ & $35 \pm 11$ & $27 \pm 6$ \\
Peak $\dot{\mathrm{VO}}{ }_{2}(\%$ pred. $)$ & $107 \pm 24$ & - \\
$\mathrm{HR} \max (\mathrm{bpm})^{*}$ & $168 \pm 17$ & $134 \pm 27$ \\
HR max $(\%$ pred.) & $92 \pm 6$ & $78 \pm 15$ \\
\hline
\end{tabular}

Data are reported as means $\pm \mathrm{SD}$. $\dot{\mathrm{V}}_{2}$ : pulmonary oxygen uptake; HR: heart rate. ${ }^{*}$ Peak $\mathrm{VO}_{2}$ in $6 \mathrm{MWT}$ corresponded to $78 \pm 13 \%$ of peak $\mathrm{VO}_{2}$ in CPET and maximum heart rate corresponded to $80 \pm 23 \%$ of that obtained in CPET.

\section{Results}

We enrolled 86 participants in this study, none of whom presented abnormal spirometric values. The general characteristics of the study sample are reported in Table 1. The frequencies of self-reported cardiovascular risk factor were $6.7 \%$ for hypertension, $3.7 \%$ for diabetes, $20.2 \%$ for dyslipidemia, $21.3 \%$ for obesity, $6.7 \%$ for smoking, and $36.0 \%$ for sedentary lifestyle.

The comparative $\mathrm{VO}_{2}$ and $\mathrm{HR}$ values achieved in both the 6MWT and CPET are reported in Table 2. The peak
$\dot{\mathrm{V}} \mathrm{O}_{2}$ in the $6 \mathrm{MWT}$ corresponded to $78 \pm 13 \%$ of that in CPET, and the maximum heart rate in the 6MWT corresponded to $80 \pm 23 \%$ of that obtained in CPET. The peak $\dot{\mathrm{VO}}_{2}$ in CPET was accurately predicted by the 6MWD $\left(\mathrm{R}^{2}=0.76\right)$ by the following linear regression equation: $\dot{\mathrm{V}} \mathrm{O}_{2}$ $\mathrm{mL} \cdot \mathrm{min}^{-1} \cdot \mathrm{kg}^{-1}=-2.863+\left(0.0563 \times 6 \mathrm{MWD}_{\mathrm{m}}\right)$ (Figure 1).

\section{Discussion}

In this study, we evaluated the intensity of 6MWT in middle-aged and older adults and derived an equation to predict the peak $\dot{\mathrm{VO}}_{2}$ achieved during CPET from the 6MWD result.

The 6MWT can be described as a moderate to highintensity exercise in which an HR of approximately $80 \%$ of maximum may occur. Kervio et al. (11) obtained similar results in 12 participants $64 \pm 1$ years of age using the same gas analyzer. The HR values reached $85 \%$ of maximum, and the $\mathrm{VO}_{2}$ peak during the third of up to five 6MWTs was quite similar to that in the present study $\left(23 \pm 1 \mathrm{~mL} \cdot \mathrm{min}^{-1} \cdot \mathrm{kg}^{-1}\right)$. The authors also showed that the peak $\dot{\mathrm{VO}}_{2}$ during the fifth $6 \mathrm{MWT}$ represented $80 \%$ of the peak $\dot{\mathrm{V}} \mathrm{O}_{2}$ obtained in CPET performed on a treadmill, compared with $78 \pm 13 \%$ in the present study. The results in patients with chronic diseases permit classification of the 6MWT as a highintensity or even a maximal test in some clinical situations (12). Although the peak $\dot{\mathrm{V}} \mathrm{O}_{2}$ during the $6 \mathrm{MWT}$ is comparable to that observed in cycle ergometer CPET, the time

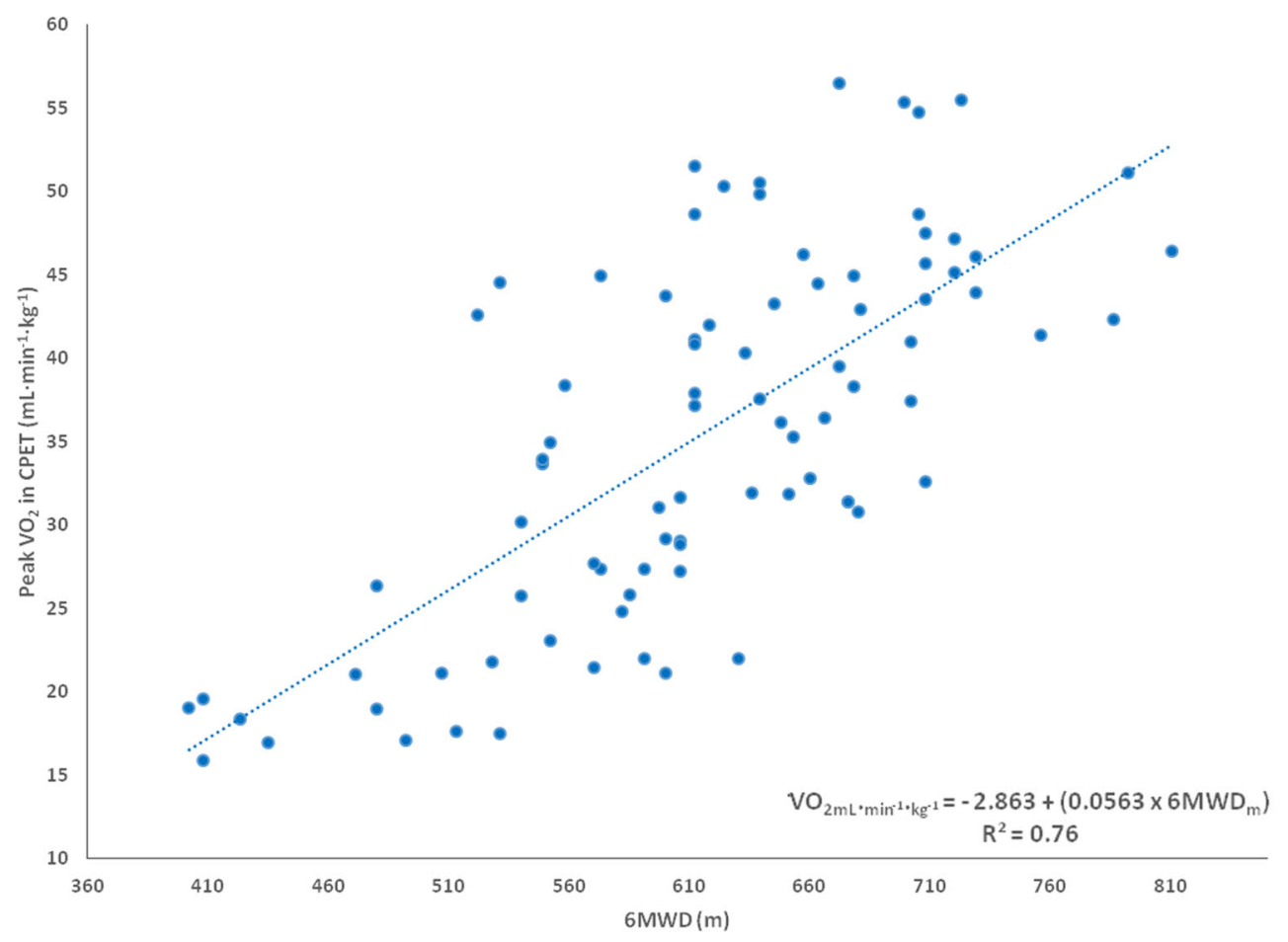

Figure 1. Significant relationship $(\mathrm{P}<0.001)$ between the 6-min walk distance $(6 \mathrm{MWD})$ and peak oxygen uptake in cardiopulmonary exercise testing (CPET). 
course of the main variables follows an exponential pattern, as was observed here and in previous studies conducted in healthy participants and in patients with chronic disease $(11,13,14)$.

Results demonstrating consistent correlations between the 6MWD and peak $\dot{\mathrm{V}} \mathrm{O}_{2}$ in CPET have been described in several previous studies $(11,12,15,16)$. Although the $6 \mathrm{MWT}$ is not a constant workload test, the walking speed reaches a constant value from the third minute of the test onwards. Such a characteristic makes the 6MWT less accurate than predicting peak $\dot{\mathrm{VO}}_{2}$ by $\mathrm{CPET}$. Despite this limitation, our prediction equation seems to be accurate, as shown in Figure 1. Several studies proposed that the 6MWD can predict $\mathrm{V}_{2}$; however, those studies involved patients with various diseases (17-19). We were able to find only one previous study that correlated peak $\dot{\mathrm{VO}}_{2}$ in $6 \mathrm{MWT}$ with peak $\dot{\mathrm{VO}}_{2}$ in CPET. Unfortunately, the authors did not perform a CPET (14). Instead, the correlation between peak $\dot{\mathrm{V}} \mathrm{O}_{2}$ in $6 \mathrm{MWT}$ and CPET was assessed using a previous reference equation for predicting peak $\dot{\mathrm{V}} \mathrm{O}_{2}$ in CPET (10). To the best of our knowledge, no study has shown such equation in a sample of healthy individuals. Nonlinear models for prediction of 6MWD have been described recently (20), but their predictive power is similar to that of linear models.

\section{References}

1. Houghton AR, Harrison M, Cowley AJ, Hampton JR. Assessing exercise capacity, quality of life and haemodynamics in heart failure: do the tests tell us the same thing? Eur J Heart Fail 2002; 4: 289-295, doi: 10.1016/S13889842(01)00236-7.

2. Maher CA, Williams MT, Olds TS. The six-minute walk test for children with cerebral palsy. Int J Rehabil Res 2008; 31: 185-188, doi: 10.1097/MRR.0b013e32830150f9.

3. Paap E, van der Net J, Helders PJ, Takken T. Physiologic response of the six-minute walk test in children with juvenile idiopathic arthritis. Arthritis Rheum 2005; 53: 351-356, doi: 10.1002/art.21175

4. Vis JC, Thoonsen H, Duffels MG, de Bruin-Bon RA, Huisman $\mathrm{SA}$, van Dijk AP, et al. Six-minute walk test in patients with Down syndrome: validity and reproducibility. Arch Phys Med Rehabil 2009; 90: 1423-1427, doi: 10.1016/j.apmr.2009.02. 015.

5. Miller MR, Hankinson J, Brusasco V, Burgos F, Casaburi R, Coates A, et al. Standardisation of spirometry. Eur Respir $J$ 2005; 26: 319-338, doi: 10.1183/09031936.05.00034805.

6. Thomas S, Reading J, Shephard RJ. Revision of the Physical Activity Readiness Questionnaire (PAR-Q). Can J Sport Sci 1992; 17: 338-345.

7. American Thoracic Society. ATS statement: guidelines for the six-minute walk test. Am J Respir Crit Care Med 2002; 166: 111-117, doi: 10.1164/ajrccm.166.1.at1102.

8. Dourado VZ, Vidotto MC, Guerra RL. Reference equations for the performance of healthy adults on field walking tests. $J$ Bras Pneumol 2011; 37: 607-614, doi: 10.1590/S1806-3713201100 0500007.
This study has some limitations. We recruited a convenience sample, but we took care to include only healthy individuals not involved in sports or vigorous physical activity. We did not find a significant influence of physical activity level on 6MWT performance. A recent study observed a significant association between the 6MWD and physical activity level (21), despite not controlling for this variable.

The 6MWT represents a high-intensity activity for the majority of middle-aged and older adults, and the strong correlation between peak $\dot{\mathrm{VO}}_{2}$ in the 6MWT and in CPET observed here shows that it is suitable for assessing aerobic functional exercise capacity in this age group. Furthermore, the $6 \mathrm{MWT}$ seems to be a valid tool for prescribing walking programs intended to improve the health of individuals in this group. The 6MWT might provide an appropriate frame of reference for the normalcy of physiological responses in adults 40 years of age and older.

\section{Acknowledgments}

Research supported by FAPESP (\#2007/08673-3). The authors would like to thank the physician Dr. Philipe Rachas Saccab for supervising a significant number of cardiopulmonary exercise tests.

9. Borg GA. Psychophysical bases of perceived exertion. Med Sci Sports Exerc 1982; 14: 377-381.

10. Wasserman K, Hansen JE, Sue DY, Stringer WW, Sietsema $\mathrm{K}$, Xing-Guo S, et al. Principles of exercise testing and interpretation. 5th edn. Philadelphia: Lippincott Williams \& Wilkins; 2011.

11. Kervio G, Carre F, Ville NS. Reliability and intensity of the sixminute walk test in healthy elderly subjects. Med Sci Sports Exerc 2003; 35: 169-174, doi: 10.1097/00005768-20030100000025.

12. Singh SJ. Walking for the assessment of patients with chronic obstructive pulmonary disease. Eur Respir Mon 2007; 40: 148-164.

13. Troosters T, Vilaro J, Rabinovich R, Casas A, Barbera JA, Rodriguez-Roisin R, et al. Physiological responses to the 6min walk test in patients with chronic obstructive pulmonary disease. Eur Respir J 2002; 20: 564-569, doi: 10.1183/ 09031936.02.02092001.

14. Hill K, Wickerson LM, Woon LJ, Abady AH, Overend TJ, Goldstein RS, et al. The 6-min walk test: responses in healthy Canadians aged 45 to 85 years. Appl Physiol Nutr Metab 2011; 36: 643-649, doi: 10.1139/h11-075.

15. Carvalho EE, Costa DC, Crescencio JC, Santi GL, Papa V, Marques $\mathrm{F}$, et al. Heart failure: comparison between sixminute walk test and cardiopulmonary test. Arq Bras Cardiol 2011; 97: 59-64, doi: 10.1590/S0066-782X2011005000056.

16. Turner SE, Eastwood PR, Cecins NM, Hillman DR, Jenkins SC. Physiologic responses to incremental and self-paced exercise in COPD: a comparison of three tests. Chest 2004; 126: 766-773, doi: 10.1378/chest.126.3.766. 
17. Cahalin L, Pappagianopoulos P, Prevost S, Wain J, Ginns L. The relationship of the 6 -min walk test to maximal oxygen consumption in transplant candidates with end-stage lung disease. Chest 1995; 108: 452-459, doi: 10.1378/chest. 108. 2.452.

18. Opasich C, Pinna GD, Mazza A, Febo O, Riccardi R, Riccardi $P G$, et al. Six-minute walking performance in patients with moderate-to-severe heart failure; is it a useful indicator in clinical practice? Eur Heart J 2001; 22: 488-496, doi: 10.1053/ euhj.2000.2310

19. Miyamoto S, Nagaya N, Satoh T, Kyotani S, Sakamaki F, Fujita M, et al. Clinical correlates and prognostic significance of six-minute walk test in patients with primary pulmonary hypertension. Comparison with cardiopulmonary exercise testing. Am J Respir Crit Care Med 2000; 161: 487-492, doi: 10.1164/ajrccm.161.2.9906015.

20. Dourado VZ, McBurnie MA. Allometric scaling of 6-min walking distance by body mass as a standardized measure of exercise capacity in healthy adults. Eur J Appl Physiol 2012; 112: 25032510, doi: 10.1007/s00421-011-2222-7.

21. Steffens D, Beckenkamp PR, Hancock M, Paiva DN, Alison JA, Menna-Barreto SS. Activity level predicts 6-minute walk distance in healthy older females: an observational study. Physiotherapy 2013; 99: 21-26, doi: 10.1016/j.physio.2011.11.004. 